\title{
Large-scale parallel server system with multi-component jobs
}

\author{
Seva Shneer \\ Heriot-Watt University \\ EH14 4AS Edinburgh, UK \\ V.Shneer@hw.ac.uk
}

\author{
Alexander L. Stolyar \\ University of Illinois at Urbana-Champaign \\ Urbana, IL 61801, USA \\ stolyar@illinois.edu
}

December 21, 2020

\begin{abstract}
A broad class of parallel server systems is considered, for which we prove the steady-state asymptotic independence of server workloads, as the number of servers goes to infinity, while the system load remains sub-critical. Arriving jobs consist of multiple components. There are multiple job classes, and each class may be of one of two types, which determines the rule according to which the job components add workloads to the servers. The model is broad enough to include as special cases some popular queueing models with redundancy, such as cancel-on-start and cancel-on-completion redundancy.

Our analysis uses mean-field process representation and the corresponding mean-field limits. In essence, our approach relies almost exclusively on three fundamental properties of the model: (a) monotonicity, (b) work conservation, (c) the property that, on average, "new arriving workload prefers to go to servers with lower workloads."
\end{abstract}

Key words and phrases: Large-scale service systems; steady-state; asymptotic independence; multi-component jobs; redundancy; replication; cancel on start; cancel on completion; load distribution and balancing

AMS 2000 Subject Classification: 90B15, 60K25

\section{Introduction}

In this paper we consider a broad class of parallel server systems, for which we prove the steady-state asymptotic independence of server workloads, as the number of servers goes to infinity, while the system load remains sub-critical. Our model is such that arriving jobs consist of multiple components. There are multiple job classes, and each class may be of one of two types. A job type determines the rule according to which the job components add workloads to the servers. The model is broad enough to include as special cases some popular queueing models with redundancy, such as cancel-on-start and cancel-on-completion redundancy.

More specifically, we consider a service system consisting of $n$ identical servers, processing work at unit rate. Jobs of multiple classes $j$ arrive according to independent Poisson processes of rate $\lambda_{j} n$. A job of each class $j$ consists of $k_{j} \geq 1$ components, while the $k_{j}$-dimensional exchangeable distribution $F_{j}$ determines the random component sizes, or workloads, $\left(\xi_{1}, \ldots, \xi_{k_{j}}\right)$. (I.i.d. component sizes is a special case of exchangeability.) Each class- $j$ job uniformly at random selects a subset of $d_{j}$ servers, $d_{j} \geq k_{j}$. Each job class may be of one of the two types, either water-filling or least-load. A job type determines the way in which the arriving job adds workload to the servers. For the least-load type the component (random) workloads $\left(\xi_{1}, \ldots, \xi_{k_{j}}\right)$ are added to the $k_{j}$ least-loaded servers out of the $d_{j}$ selected by the job. For the water-filling type we describe the workload placement algorithm via the following illustration. Suppose, $d_{j}=4, k_{j}=2$, the component sizes realization is $(10,5)$, and the workloads of the selected 4 servers are $5,12,7,16$. Then, adding 10 units of the 
first component workload in the water-filling fashion brings the selected servers' workloads to 11,12,11, 16 . Before we place the next - second - component's workload, we exclude one of the selected servers that currently have the smallest workload - it will be one of the servers with workload 11 in this illustration. Then, placing the second component's workload 5 in the water-filling fashion on the remaining 3 selected servers, brings the servers' workloads to $11,14,14,16$. In general, after each component is placed, the set of selected servers is reduced by excluding one of the servers with the smallest workload.

We assume that the system is sub-critically loaded, $\sum_{j} \lambda_{j} s_{j}<1$, where $s_{j}$ is the total expected workload brought by a class $j$ job. It is not hard to see that the system is stable for each $n$. Our main results, Theorem 1 and Corollary 2, prove the steady-state asymptotic independence property: for any fixed integer $m \geq 1$, as $n \rightarrow \infty$, the steady-state workloads of a fixed set of $m$ servers (say, servers $1, \ldots, m$ ), become asymptotically independent. This property, in addition to be important in itself, in many cases allows one to obtain asymptotically exact system performance metrics, such as steady-state job delay mean or distribution.

Our model is related to - but not limited to - queueing models with cancel-on-start and cancel-on-completion redundancy $1,3,7,8,12,15]$. In the redundancy models each job places its "replicas" on a selected subset of servers. The replicas may be served by their servers simultaneously. When a certain number of the job replicas start [resp., complete] their service, all other replicas are "canceled" and removed from the system. Hence the term cancel-on-start [resp., cancel-on-completion]. We postpone until the next section the detailed discussion of our model, including its relations to the models with redundancy. At this point we note that our least-load job type covers the cancel-on-start redundancy, and our water-filling job type covers the cancelon-completion redundancy in the special case of i.i.d. exponentially distributed replica sizes. Moreover, our model allows multiple job classes, of different types. We also note that, for example, the model in 7 and some of the models in 10 are special cases of ours; the steady-state asymptotic independence was used in those papers as a conjecture; our Corollary 2 proves this conjecture (for those models in particular), thus formally substantiating the asymptotic steady-state performance metrics derived for those models in $[7$ and 10 .

Methodologically, this paper belongs to the line of work establishing the steady-state asymptotic independence in different contexts, e.g. [5 $13,14,16]$. Our approach is based on analyzing the mean-field (fluid) scaled process and its limit. One part of our analysis, namely establishing asymptotic independence of server workloads over a finite time interval, closely follows the previous work, namely [5, 9. But, the main part of the analysis, namely the transition from the finite-interval asymptotic independence to the steady-state asymptotic independence, is different from that in [5]. (Paper [9] does not have a steady-state asymptotic independence result.) Specifically, we rely on the dynamics - transient behavior - of the mean-field scaled process and its limit; in this sense, our approach is close to that in [13 14. (The approach of [5] relies in an essential way on the direct steady-state estimates of the marginal workload distributions, obtained in [4].) At a high level, one may say that our approach relies almost exclusively on three fundamental properties of the model: (a) monotonicity, (b) work conservation, (c) the property that, on average, "new arriving workload prefers to go to servers with lower workload." Consequently, we believe this approach for establishing the steady-state asymptotic independence may apply more broadly, to other models as well, as long as they possess these three fundamental properties.

The rest of the paper is organized as follows. A more detailed discussion of our model and results is given in Section 2, which is followed by a brief review of previous work in Section 3 . Section 4 gives basic notation used throughout the paper. Section 5 presents our formal model and the main results, Theorem 1 and Corollary 2. In Section 6 we define some generalizations of our model and give their basic properties; these generalizations, while may be of independent interest, are primarily for the purposes of the analysis of our original model. Section 7 contains more auxiliary facts used in the analysis. Section 8 gives results on the finite-interval asymptotic independence of the server workloads. In Section 9 we define limits of the mean-field (fluid) scaled processes; we call these limits fluid sample paths (FSP). In Section 10 we study properties of the FSPs, starting specifically from "empty" initial state. In Section 11 we define and study the FSP fixed point, which is the point to which the FSP trajectory, starting from the empty state, converges. Finally, Section 12 contains the proof of the main result (Theorem 11), which employs the results developed 
in Sections 6 11

\section{Discussion of the model and main results}

The least-load job type is motivated by two scenarios. First, if we consider a system such that the current server workloads can be observed on a subset of $d_{j}$ selected servers, and a the job consists of $k_{j}$ components, it directly makes sense to place those components for service on the least-loaded $k_{j}$ of those $d_{j}$ servers. (See e.g. LL(d) policy in [5], which is a special case of our model with a single least-load class with $d_{j}=d$, $k_{j}=1$.) The second scenario arises in systems where the current workloads are not observable, and which use redundancy to improve performance. (See e.g. 12 for a general motivation for redundancy.) For example, suppose a class- $j$ job places $d_{j}$ "replicas" on $d_{j}$ randomly selected servers, and each server processes its work (replicas of different jobs) in the First-Come-First-Serve (FCFS) order. Suppose the job, to be completed, requires only $k_{j}$ (out of $d_{j}$ ) replicas to be processed, and as soon as the first $k_{j}$ replicas of the job start being processed, the remaining $d_{j}-k_{j}$ replicas of the job are "canceled" and immediately removed from their corresponding servers. This is usually referred to as cancel-on-start redundancy. (See e.g. 2, 3], where a special case $k_{j}=1$ is considered.) We will call it $\left(d_{j}, k_{j}\right)$-c.o.s. redundancy, where $\left(d_{j}, k_{j}\right)$ are the parameters of class $j$. Clearly, from the point of view of the server workload evolution (which need not be observable in this case), the described $\left(d_{j}, k_{j}\right)$-c.o.s. redundancy is equivalent to simply placing $k_{j}$ job replicas on the $k_{j}$ least loaded (out of $d_{j}$ ) servers, and not placing any workload on the remaining $d_{j}-k_{j}$ servers. Thus, a job class using $\left(d_{j}, k_{j}\right)$-c.o.s. redundancy can be equivalently viewed as a least-load job class in terms of our model, with the $k_{j}$ job components being the first $k_{j}$ replicas.

The water-filling job type motivation is also two-fold. First, suppose a job class $j$ is of the water-filling type, with $k_{j}=1$. Consider a class $j$ job; it has one component. Suppose further that this component's workload can be arbitrarily divided between servers, in the sense that a parallel processing by multiple servers is allowed. (For example, the servers may represent different data transmission channels, with a job being a file that needs to be transmitted, and the job size being the file size.) Suppose the job can use $d_{j}$ randomly selected servers. The servers process workload in the FCFS order. The job is completed when all its workload is processed. Then, if the objective is to minimize the job completion time, its workload should be placed into the selected $d_{j}$ servers in the water-filling fashion. This can be done directly, if the workloads of the selected $d_{j}$ servers and the job workload are observable, or indirectly, as follows. The job joins the FCFS queues at each of the selected servers. When this job, at any of the selected servers, reaches the head of the queue - i.e., can start using that server - that server starts processing the job, possibly in parallel with other selected servers. The job is completed when the total amount of service it receives from all servers is equal to its size, at which point the job is removed from all queues. From the point of view of the server workload evolution (which need not be observable in this case), the described procedure is equivalent to simply placing the job's single component on $d_{j}$ selected servers in the water-filling fashion.

The second motivation for the water-filling job type arises from cancel-on-completion redundancy ( $[1,2,7,8$, 10 12 15 ). Suppose a class- $j$ job places $d_{j}$ job replicas on $d_{j}$ randomly selected servers. Each server processes its work (replicas of different jobs) in the FCFS order. Suppose the job, to be completed, requires only $k_{j}$ (out of $d_{j}$ ) replicas to be processed, and as soon as the first $k_{j}$ replicas of the job complete their service, the remaining $d_{j}-k_{j}$ replicas of the job are "canceled" and immediately removed from their corresponding servers. (Hence the name cancel-on-completion.) We will call this $\left(d_{j}, k_{j}\right)$-c.o.c. redundancy, where $\left(d_{j}, k_{j}\right)$ are the parameters of class $j$. Suppose, in addition, that the replica sizes for a class-j job are i.i.d. exponential random variables with mean $s_{j} / k_{j}$. (This additional assumption, as well as the further assumption that $k_{j}=1$, is used, e.g., in 7].) Under this additional assumption (of i.i.d. exponential replica sizes), it is easy to observe that, from the point of view of the servers' workload evolution (which need not be observable), the described $\left(d_{j}, k_{j}\right)$-c.o.c. redundancy is equivalent to placing on the selected $d_{j}$ servers a water-filling job with the following parameters: $\left(d_{j}, k_{j}\right)$ are the same as above, and the component sizes are i.i.d. exponential random variables with mean $s_{j} / k_{j}$. Indeed, the job component 1 places (stochastically) exactly the same amounts of additional workload on the servers as the workloads placed by all replicas up to the time of 
the first replica service completion. Similarly, the job component 2 places (stochastically) exactly the same amounts of additional workload on the servers as the workloads placed by all replicas from the time of the first replica service completion until the time of the second replica service completion. And so on. Thus, a job class using $\left(d_{j}, k_{j}\right)$-c.o.c. redundancy (under the additional assumption of i.i.d. exponential replica sizes) can be equivalently viewed as a water-filling job class in terms of our model, with parameters $\left(d_{j}, k_{j}\right)$ and i.i.d. exponentially distributed components.

We see that our model is very broad. In 5 it is proved (among other results) that the steady-state asymptotic independence (our Corollary 2 holds for the special case of a single, least-load job class with $k_{j}=1$. (See LL(d) model in [5].) The (d,1)-c.o.c. model with i.i.d. exponential replica sizes, considered in 7], is a special case of our model, with a single, water-filling job class, with $k_{j}=1$ and i.i.d. exponential component sizes. One of the models considered in 10 (called LL $(\mathrm{d}, \mathrm{k}, 0)$ there) is a special case of ours, with a single least-load job class. In both 7$]$ and $[10$ the steady-state asymptotic independence was used as a conjecture; our Corollary 2 proves this conjecture for both models. Furthermore, since our model allows multiple job classes of different types, Corollary 2 establishes the steady-state asymptotic independence, for example, for a system with two job classes - one as in 7 and one as the $\mathrm{LL}(\mathrm{d}, \mathrm{k}, 0)$ class in 10 .

\section{Previous work}

The work on the steady-state asymptotic independence in the large-scale regime, with the number of servers and the arrival rate increasing to infinity, while the system load remains sub-critical, includes, e.g., papers 5, 13, 16. Papers [5, 16] prove this for the celebrated "power-of-d" choices algorithm, where each arriving (single-component) job joins the shortest queue out of $d$ randomly selected; 16] does this for the exponentially distributed job sizes, while [5] extends the results to more general job size distributions, namely those with decreasing hazard rate (DHR). Note that a standard power-of-d choices algorithm is not within the framework of our model, because job placement decisions depend on the queue lengths (numbers of jobs), as opposed to depending on the workloads. However, [5] also considers - and establishes the steady-state asymptotic independence for - the LL(d) model, which is a special case of our model with the single, least-load job class with $d_{j}=d$ and $k_{j}=1$. Note that, equivalently, this is the single-class $(d, 1)$-c.o.s. redundancy model. The main results of 5 in turn rely on the uniform estimates of the marginal stationary distribution of a single server state, obtained in [4]. Paper [13] proves the steady-state asymptotic independence under a pull-based algorithm, also for the model with single-component jobs, having DHR size distributions. (The model in [13 is also not within the framework of the present paper model.)

For the redundancy models, such as in $[1,3,7,8,10,12,15]$, we are not aware of any prior steady-state asymptotic independence results, besides the already mentioned $(d, 1)$-c.o.s. result in [5]. However, the steady-state asymptotic independence conjecture is often used (e.g. 7, 10, 15]) to obtain estimates of the steady-state performance metrics of large scale systems.

Paper 15 introduces redundancy as a way to reduce job delays. It considers the $(d, 1)$-c.o.c. redundancy model, with generally distributed replica sizes. (As such, this model is not within the framework of our model.) The paper uses the steady-state asymptotic independence conjecture to estimate the average job delay when the system is large.

Paper 12 introduces and motivates the $(d, k)$-c.o.c. redundancy model, and establishes a variety of monotonicity properties of the average job delay with respect to the selection set size $d$, under different assumptions on the replica size distribution. Some of the results of $[12$ are for the $(d, k)$-c.o.c. redundancy model with i.i.d. exponential replica sizes, which is a special case of our model, but 12 does not consider the asymptotic regime with $n \rightarrow \infty$.

As already described earlier, paper 7] studies the $(d, 1)$-c.o.c. redundancy model with i.i.d. exponential replica sizes, and obtains asymptotically exact expressions for the job delay distribution, based on the steady-state asymptotic independence conjecture. Our results prove this conjecture, thus completing formal 
substantiation of those asymptotic expressions.

Paper [10] studies general redundancy models - more general than c.o.s. and c.o.c. that we described earlier - and uses the steady-state asymptotic independence conjecture to characterize and compute steady-state performance metrics. Some (not all) of the redundancy schemes in 10 are within our model framework. For example, $\mathrm{LL}(\mathrm{d}, \mathrm{k}, 0)$ redundancy in 10 is a special case of our model with a single least-load class. Thus, again, by proving the steady-state asymptotic independence, our results complete formal substantiation of some of the asymptotic results in 10 .

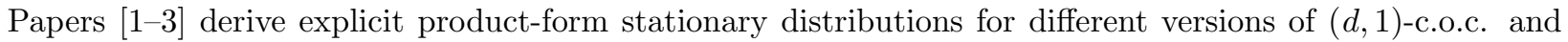
$(d, 1)$-c.o.s. redundancy, assuming i.i.d. exponential replica sizes.

\section{Basic notation}

We denote by $\mathbb{R}$ and $\mathbb{R}_{+}$the sets of real and real non-negative numbers, respectively, and by $\mathbb{R}^{n}$ and $\mathbb{R}_{+}^{n}$ the corresponding $n$-dimensional product sets. By $\overline{\mathbb{R}}_{+} \doteq \mathbb{R}_{+} \cup\{\infty\}$ we denote the one-point compactification of $\mathbb{R}_{+}$, where $\infty$ is the point at infinity, with the natural topology. We say that a function is RCLL if it is right-continuous with left-limits. Inequalities applied to vectors [resp. functions] are understood component-wise [resp. for every value of the argument]. The sup-norm of a scalar function $f(w)$ is denoted $\|f(\cdot)\| \doteq \sup _{w}|f(w)|$; the corresponding convergence is denoted by $\stackrel{u}{\rightarrow}$. U.o.c. convergence means uniform on compact sets convergence, and is denoted by $\stackrel{\text { u.o.c. }}{\rightarrow}$. We use notation: $a \vee b \doteq \max \{a, b\}, a \wedge b \doteq \min \{a, b\}$. Abbreviation WLOG means without loss of generality.

For a random process $Y(t), t \geq 0$, we denote by $Y(\infty)$ the random value of $Y(t)$ in a stationary regime (which will be clear from the context). Symbol $\Rightarrow$ signifies convergence of random elements in distribution; $\stackrel{P}{\rightarrow}$ means convergence in probability. W.p.1 means with probability one. I.i.d. means independent identically distributed. Indicator of event or condition $B$ is denoted by $\mathbf{I}(B)$. If $X, Y$ are random elements taking values in set $\mathcal{X}$, on which a partial order $\leq$ is defined, then the stochastic order $X \leq_{s t} Y$ means that $X$ and $Y$ can be coupled (constructed on a common probability space) so that $X \leq Y$ w.p.1.

We will use the following non-standard notation. Suppose $f_{w}^{n}, n \rightarrow \infty$, is a sequence of random functions of $w$, and $f_{w}$ is a deterministic function of $w$. Then, for a fixed $w$,

$$
(P) \liminf _{n \rightarrow \infty} f_{w}^{n} \geq f_{w} \quad \text { means } \quad\left[\left(f_{w}^{n}-f_{w}\right) \wedge 0\right] \stackrel{P}{\rightarrow} 0, n \rightarrow \infty
$$

and for a subset $A$ of the domain of $w$,

$$
(P) \liminf _{n \rightarrow \infty}\left(f_{w}^{n}, w \in A\right) \geq\left(f_{w}, w \in A\right) \quad \text { means } \quad \inf _{w \in A}\left[\left(f_{w}^{n}-f_{w}\right) \wedge 0\right] \stackrel{P}{\rightarrow} 0, n \rightarrow \infty
$$

Analogously,

$$
\begin{gathered}
(P) \limsup _{n \rightarrow \infty} f_{w}^{n} \leq f_{w} \quad \text { means } \quad\left[\left(f_{w}^{n}-f_{w}\right) \vee 0\right] \stackrel{P}{\rightarrow} 0, n \rightarrow \infty \\
(P) \limsup _{n \rightarrow \infty}\left(f_{w}^{n}, w \in A\right) \leq\left(f_{w}, w \in A\right) \quad \text { means } \quad \sup _{w \in A}\left[\left(f_{w}^{n}-f_{w}\right) \vee 0\right] \stackrel{P}{\rightarrow} 0, n \rightarrow \infty \\
(P) \lim _{n \rightarrow \infty} f_{w}^{n}=f_{w} \quad \text { means } \quad f_{w}^{n} \stackrel{P}{\rightarrow} f_{w}, n \rightarrow \infty \\
(P) \lim _{n \rightarrow \infty}\left(f_{w}^{n}, w \in A\right)=\left(f_{w}, w \in A\right) \quad \text { means } \sup _{w \in A}\left|f_{w}^{n}-f_{w}\right| \stackrel{P}{\rightarrow} 0, n \rightarrow \infty
\end{gathered}
$$




\section{$5 \quad$ Formal model and main results}

\section{$5.1 \quad$ Model}

There are $n$ identical servers. The unfinished work of a server at a given time will be referred to as its workload. Each server processes its workload at rate 1 . There is a finite set $\mathcal{J}$ of job classes, which are indexed by $j$. (Set $\mathcal{J}$ does not depend on $n$.) Jobs of class $j$ arrive according to a Poisson process of rate $\lambda_{j}(n) n$. Associated with each class $j$ there are three parameters: integers $k_{j}$ and $d_{j}$ such that $1 \leq k_{j} \leq d_{j}$, and the exchangeable probability distribution $F_{j}$ on $\mathbb{R}_{+}^{k_{j}}$. A class- $j$ job consists of $k_{j}$ components, with each component having a (random) size (which is the amount of new workload this component brings); $F_{j}$ is the joint distribution of random component sizes $\left(\xi_{1}^{(j)}, \ldots, \xi_{k_{j}}^{(j)}\right)$ for a class- $j$ job. Exchangeability of $F_{j}$ means that it is invariant w.r.t. permutations of component indices. We assume that $s_{j} \doteq \mathbb{E} \sum_{\ell} \xi_{\ell}^{(j)}=k_{j} \mathbb{E} \xi_{1}^{(j)}<\infty$. WLOG, we can and do assume that $s_{j}>0$ for each class $j$. We will denote $d \doteq \max _{j} d_{j}$.

Each job class $j$ may be of one of the two types, either water-filling or least-load. The corresponding nonintersecting subsets of $\mathcal{J}$ we denote by $\mathcal{J}_{w f}$ and $\mathcal{J}_{l l}$. (Either of them may be empty.) A job type determines the way in which the arriving job adds workload to the servers. We will describe the job types separately.

A least-load job class $j \in \mathcal{J}_{l l}$. When such a job arrives, $d_{j}$ servers are selected uniformly at random; these servers form the selection set of the job. Then $k_{j}$ of the selected servers, that are least-loaded (have the smallest workload), are picked; the workload ties are broken in an arbitrary fashion. Then, independently of the process history, random component sizes $\left(\xi_{1}, \ldots, \xi_{k_{j}}\right)$ are drawn according to distribution $F_{j}$. Then, workload $\xi_{1}$ is added to the least-loaded of those servers, $\xi_{2}$ is added to the second least-loaded of those servers, and so on.

A water-filling job class $j \in \mathcal{J}_{w f}$. When such a job arrives, its selection set of $d_{j}$ servers is selected uniformly at random. Then, independently of the process history, random component sizes $\left(\xi_{1}, \ldots, \xi_{k_{j}}\right)$ are drawn according to distribution $F_{j}$. We "take" the first component, and place its $\xi_{1}$-size workload on the servers within the selection set in the "water-filling" fashion. (For example, suppose the selection set consists of 4 servers, $1,2,3,4$, with workloads $W_{1}=5, W_{2}=12, W_{3}=7, W_{4}=16$, and suppose $\xi_{1}=10$. Then, adding the workload of size 10 to these servers in the water-filling fashion will result in the new workloads being $W_{1}=11, W_{2}=12, W_{3}=11, W_{4}=16$. That is servers 1 and 3 will receive non-zero additional workloads, 6 and 4, respectively, and will end up with equal workload 11. Servers 2 and 4 will not receive any of the first component's workload.) After this, there will be one or more selected servers that currently have the smallest workload. (Servers 1 and 3 in the illustration above.) Let us call them component-1 servers. Then we pick one of the component-1 servers (in an arbitrary fashion), and exclude it from further workload placement by this job. Then, we "take" the second component, and place its $\xi_{2}$-size workload on the remaining $d_{j}-1$ servers by continuing the water-filling. The servers that will have the smallest workload after that we call component-2 servers. Then we exclude one of the component-2 servers, and so on, until the workload of all $k_{j}$ components is placed. (Note that we could define an additional - different - water-filling type, such that the water-filling continues to use all $d_{j}$ selected servers, without excluding one of the servers after each component placement. This, however, is just a special case of the type we just defined, with $k_{j}$ components replaced by the single component of the size $\sum_{i} \xi_{i}$.)

By the model definition, for each class $j$, regardless of its type, the total expected additional workload it brings to the system is equal to $s_{j}$.

\section{$5.2 \quad$ Asymptotic regime. Mean-field scaled process}

We consider the sequence of systems with $n \rightarrow \infty$, and assume

$$
\lambda_{j}(n) \rightarrow \lambda_{j}>0, \quad j \in \mathcal{J}
$$


Further assume that the system is (asymptotically) sub-critically loaded

$$
\rho \doteq \sum_{j} \lambda_{j} s_{j}<1
$$

Denote the (limiting) total job arrival rate per server by

$$
\lambda \doteq \sum_{j} \lambda_{j}
$$

WLOG, we can and will assume $\lambda=\rho$. (We can achieve this by rescaling time, if necessary.)

To improve paper readability, let us assume that $\lambda_{j}(n)=\lambda_{j}$ for each $n$. Having converging $\lambda_{j}(n)$ does not change anything of substance, but clogs exposition. (However, we do need and will use the fact that our results hold for converging arrival rates.) Similarly, throughout the paper, we will often consider "an servers" for some real $a$, ignoring the fact that an may be non-integer; it would be more precise to consider, for example, "an-rounded-up servers," but it would just clog the exposition, rather than creating any difficulties.

From now on, the upper index $n$ of a variable/quantity will indicate that it pertains to the system with $n$ servers, or $n$-th system. Let $W_{i}^{n}(t)$ denote the workload of server $i$ at time $t$ in the $n$-th system. (When $W_{i}^{n}(t)=0$ we say that server $i$ at time $t$ is empty.) Consider the following mean-field, or fluid, scaled quantities:

$$
x_{w}^{n}(t) \doteq(1 / n) \sum_{i} \mathbf{I}\left\{W_{i}^{n}(t)>w\right\}, \quad w \geq 0 .
$$

That is, $x_{w}^{n}(t)$ is the fraction of servers $i$ with $W_{i}^{n}(t)>w$. Then $x^{n}(t)=\left(x_{w}^{n}(t), w \geq 0\right)$ is the system state at time $t$; note that $x_{0}^{n}(t)$ is the fraction of busy servers (the instantaneous system load).

For any $n$, the state space of the process $\left(x^{n}(t), t \geq 0\right.$ ) is a subset of a common (for all $n$ ) state space $\mathcal{X}$, whose elements $x=\left(x_{w}, w \geq 0\right)$ are non-increasing RCLL functions of $w$, with values $x_{w} \in[0,1]$. An element $x \in \mathcal{X}$ defines a probability measure on $\overline{\mathbb{R}}_{+}$, with $1-x_{w}$ being the measure of $[0, w]$ for $0 \leq w<\infty$. Denote $x_{\infty} \doteq \lim _{w \rightarrow \infty} x_{w}$; then $x_{\infty}$ is the measure of $\{\infty\}$. An element $x \in \mathcal{X}$ we will call proper, if $x_{\infty}=0$, i.e. if the corresponding probability measure is concentrated on $\mathbb{R}_{+}$. We will equip the space $\mathcal{X}$ with the topology of weak convergence of measures on $\overline{\mathbb{R}}_{+}$; equivalently, $y \rightarrow x$ if and only if $y_{w} \rightarrow x_{w}$ for each $0<w<\infty$ where $x$ is continuous. We also can and do equip $\mathcal{X}$ with a metric consistent with the topology. Obviously, $\mathcal{X}$ is compact.

For any $n$, process $x^{n}(t), t \geq 0$, is Markov with state space $\mathcal{X}$, and with sample paths being RCLL functions of $t \geq 0$. Moreover, this is a renewal process, with renewals occurring when all servers become empty.

Under the subcriticality assumption (3), i.e. $\rho=\lambda<1$, the stability (positive Harris recurrence) of the process $\left(x^{n}(t), t \geq 0\right.$ ), for any $n$, is not hard to establish. (Positive recurrence in this case simply means that the expected time to return to the empty state is finite.) It can be established, for example, using the fluid limit technique, analogously to the way it is done in [6]. The key property that the fluid limit for our model shares with that in 6 is that if there is a subset of servers, whose fluid workloads are greater than in the rest of the servers, the average per-server rate at which the servers within the subset will receive new workload is at most $\rho<1$. (See $\sqrt{12}$ ) in Section 6.2) We do not provide further details of the stability proof.

Given that the process $\left(x^{n}(t), t \geq 0\right)$ is stable, it has a unique stationary distribution. Let $x^{n}(\infty)$ be a random element whose distribution is the stationary distribution of the process; in other words, this is a random system state in the stationary regime.

\subsection{Main results}

Theorem 1. There exists a unique proper element $x^{*} \in \mathcal{X}$, with $x_{0}^{*}=\lambda=\rho$, such that

$$
x^{n}(\infty) \Rightarrow x^{*}, \quad n \rightarrow \infty .
$$


Function $x_{w}^{*}, w \geq 0$, is Lipschitz continuous and strictly decreasing (and then everywhere positive).

Corollary 2 (Steady-state asymptotic independence). For any fixed integer $m \geq 1$, the following holds. For each $n$, let $\left[W_{1}^{n}(\infty), \ldots, W_{m}^{n}(\infty)\right]$ denote the random value of $\left[W_{1}^{n}(t), \ldots, W_{m}^{n}(t)\right]$ in the stationary regime. Then

$$
\left[W_{1}^{n}(\infty), \ldots, W_{m}^{n}(\infty)\right] \Rightarrow\left[W_{1}^{*}, \ldots, W_{m}^{*}\right], \quad n \rightarrow \infty
$$

where random variables $W_{i}^{*}$ are i.i.d., with $\mathbb{P}\left\{W_{i}^{*}>w\right\}=x_{w}^{*}, w \geq 0$.

Corollary 2 follows from Theorem 1 and the symmetry between (exchangeability of) servers. Indeed, by the symmetry, the distribution of $\left[W_{1}^{n}(\infty), \ldots, W_{m}^{n}(\infty)\right]$ is equal to the joint distribution of workloads of $m$ servers chosen uniformly at random. Since $m$ is fixed and as $n \rightarrow \infty$ the steady-state empirical measure $x^{n}(\infty)$ of the server workloads converges to the deterministic element $x^{*}$, the statement easily follows.

\section{$6 \quad$ More general systems}

\subsection{Infinite workloads and truncation. Monotonicity properties.}

For the purposes of our analysis, it will be convenient to consider two generalizations of our model. (These more general systems may be of independent interest as well.)

First, we generalize our original system defined above, by allowing some of the servers to have an infinite workload. Specifically, if server $i$ workload is initially infinite, $W_{i}^{n}(0)=\infty$, then, by convention, it remains infinite at all times, $W_{i}^{n}(t)=\infty, t \geq 0$. The same workload placement rules apply even if some server workloads are infinite, with the convention that an infinite workload remains infinite when "more" workload is added to it. Note that if one or more server workloads are initially infinite, this implies that $x_{\infty}^{n}(0)>0$ and $x_{\infty}^{n}(t)=x_{\infty}^{n}(0)$ for all $t \geq 0$.

A second convenient generalization is a system, where the workload of the servers is truncated at some level $c$, where $0 \leq c \leq \infty$. Such a truncated system is defined exactly as the original one, except when an arriving job adds workload to servers, each server's workload is capped (truncated) at level $c$ every time the algorithm would increase it above $c$. The workload lost due to truncation is removed from the system. The case $c=\infty$ corresponds to the original, non-truncated system, where the arriving workload is never lost. Note that, if $c<\infty$, then the stability for any $n$ (and any $\lambda$ ) is automatic. The process corresponding to the truncated system with parameter $c$, we denote by $x^{n, c}(t), t \geq 0$; if superscript $c$ is absent, this corresponds to $c=\infty$, i.e. the process $x^{n}(\cdot)$ is for the original non-truncated system.

Finally, if the process starts specifically from the "empty" initial state (with all servers having zero initial workload), we will add superscript $\emptyset$ to the process notation: $x^{n, c, \emptyset}(t), t \geq 0$; therefore, $x_{0}^{n, c, \emptyset}(0)=0$. So, for example, $x^{n, \emptyset}(\cdot)$ denotes the original non-truncated process, starting from the empty state.

The analysis in this paper relies on the system monotonicity, and related properties. We will need several such properties. They are all related and rather simple.

Lemma 3. Consider two versions of the process, $x^{n, c}(\cdot)$ and $\hat{x}^{n, \hat{c}}(\cdot)$, such that $x^{n, c}(0) \leq \hat{x}^{n, \hat{c}}(0), c \leq \hat{c}$. Then these processes can be coupled so that, w.p.1,

$$
x^{n, c}(t) \leq \hat{x}^{n, \hat{c}}(t), \forall t \geq 0 .
$$

Furthermore, if the process $\hat{x}^{n, \hat{c}}(\cdot)$ is modified so that, in addition to the job arrival process (as defined in our model), arbitrary amounts of workload may be added at arbitrary times to arbitrary servers, the property (8) still holds.

Proof. As far as the mean-field scaled processes $x^{n, c}(\cdot)$ and $\hat{x}^{n, \hat{c}}(\cdot)$ are concerned, WLOG, we can assume that, after each job arrival and/or other workload addition(s), the actual servers $1, \ldots, n$ are relabeled, so 
that the workloads $W_{1}^{n}, \ldots, W_{n}^{n}$ are non-decreasing. Then, for the two processes it is sufficient to couple in the natural way the arrival processes and the job selection sets, to see that (8) must prevail at all times.

From Lemma 3 , we obtain the following

Corollary 4. For any $0 \leq c \leq \infty$, the process $x^{n, c, \emptyset}(\cdot)$ is monotone in time $t \geq 0$, namely

$$
x^{n, c, \emptyset}\left(t_{1}\right) \leq{ }_{s t} x^{n, c, \emptyset}\left(t_{2}\right), \quad \forall t_{1} \leq t_{2} .
$$

Lemma 5. Consider two versions of the process, $x^{n, c}(\cdot)$ and $\hat{x}^{n, \hat{c}}(\cdot)$, such that $c, \hat{c} \in[0, \infty]$. Suppose that for some fixed $w \in[0, c \wedge \hat{c}]$, we have $x_{v}^{n, c}(0)=\hat{x}_{v}^{n, \hat{c}}(0)$ for $0 \leq v \leq w$. Then these processes can be coupled so that, w.p.1, for $t \in[0, w]$ and $v \in[0, w-t]$,

$$
x_{v}^{n, c}(t)=\hat{x}_{v}^{n, \hat{c}}(t) .
$$

Proof. We couple the two processes in the natural way, as in the proof of Lemma 3 . The proof then follows by induction on the times of job arrivals in the interval $[0, w]$. Indeed, if $t_{1} \leq w$ in the time of the first job arrival, 99 of course holds for all $t \in\left[0, t_{1}\right)$. It is then easy to see that the changes of $x_{v}^{n, c}$ and $\hat{x}_{v}^{n, \hat{c}}$ for $v \leq w-t_{1}$, at time $t_{1}$, only depend on those servers with workloads at most $w-t_{1}$, which are the same for both systems; we also observe that if any of those servers changes its workload to a value not exceeding $w-t_{1}$, the change will be exactly same in both systems. Then 9 holds for $t=t_{1}$. Then, (9) holds until the time $t_{2}$ of the second job arrival or $w$, whichever is smaller. And so on.

Lemma 5 and Lemma 3 imply the following more general form of Lemma 5.

Lemma 6. Consider two versions of the process, $x^{n, c}(\cdot)$ and $\hat{x}^{n, \hat{c}}(\cdot)$, such that $c, \hat{c} \in[0, \infty]$. Suppose that for some fixed $w \in[0, c \wedge \hat{c}]$, we have $x_{v}^{n, c}(0) \leq \hat{x}_{v}^{n, \hat{c}}(0)$ for $0 \leq v \leq w$. Then these processes can be coupled so that, w.p.1, for $t \in[0, w]$ and $v \in[0, w-t]$,

$$
x_{v}^{n, c}(t) \leq \hat{x}_{v}^{n, \hat{c}}(t) .
$$

\subsection{Equivalent representation of a system with some workloads being infinite.}

Let $b \in(0,1]$ be fixed. For each $n$, consider the system with the initial state such that $(1-b) n$ servers have infinite workloads, while the remaining $b n$ servers' workloads are finite. Let $B=B(n)$ denote the set of servers with finite workloads. Then, for each $n$, the evolution of the subsystem consisting of servers in $B-$ let us call it $B$-subsystem - can be equivalently described as follows. The number of servers is $b n$. Each job class $j$ "breaks down" into multiple classes $(j, m), m=1, \ldots, d_{j}$, as follows. Let $\pi_{j, m}(n)$ be the probability that exactly $m$ servers selected by a class $j$ job, will be in $B$. Note that

$$
\sum_{m} \pi_{j, m}(n) m=b d_{j}
$$

Then, for a given $n$, class $(j, m)$ in the $B$-system has the following parameters: arrival rate per server $\lambda_{j, m}(n)=\lambda_{j} \pi_{j, m}(n) / b, d_{j, m}=m, k_{j, m}=k_{j} \wedge m$, the distribution $F_{j, m}$ of the component sizes is the projection of the distribution $F_{j}$ on the first $m$ components. $\left(d_{j, m}, k_{j, m}\right.$ and $F_{j, m}$ do not depend on $n$.) Clearly, as far as evolution of the $B$-system is concerned, this new description is consistent with the actual behavior. The load of the $B$-system is

$$
\rho_{B}(n)=\sum_{j, m} \lambda_{j, m}(n) s_{j}\left(k_{j, m} / k_{j}\right) .
$$

Recall that the load of the original system, for any $n$, is $\rho=\sum_{j} \lambda_{j} s_{j}$.

The following fact is very intuitive - by the nature of the workload placement algorithm, the arriving workload "prefers" servers with finite workloads. 
Lemma 7. For each n,

$$
\rho_{B}(n) \geq \rho
$$

Proof. We can write:

$$
\rho=\sum_{j} \lambda_{j} s_{j}=\sum_{j} \sum_{m} \frac{\lambda_{j} \pi_{j, m}(n)}{b} \frac{m}{d_{j}} s_{j} \leq \sum_{j} \sum_{m} \frac{\lambda_{j} \pi_{j, m}(n)}{b} \frac{m \wedge k_{j}}{d_{j} \wedge k_{j}} s_{j}=\sum_{j} \sum_{m} \lambda_{j, m}(n) \frac{k_{j, m}}{k_{j}} s_{j}=\rho_{B}(n) .
$$

Note that, if $\rho_{A}(n)$ is the load of the complementary subsystem, consisting of the $(1-b) n$ infinite-workload servers, then $\rho=b \rho_{B}(n)+(1-b) \rho_{A}(n)$ and, therefore,

$$
\rho_{A}(n) \leq \rho .
$$

Consider now a sequence of the above systems, with $n \rightarrow \infty$. Recall that the number of servers in the $B$-system is $b n$. Note that

$$
\lim _{n} \pi_{j, m}(n)=\pi_{j, m}=\frac{d_{j} !}{m !\left(d_{j}-m\right) !} b^{m}(1-b)^{d_{j}-m} .
$$

Then,

$$
\lambda_{j, m}(n) \rightarrow \lambda_{j, m}=\lambda_{j} \pi_{j, m} / b,
$$

and the $B$-subsystem (limiting) load is

$$
\rho_{B}=\lim _{n} \rho_{B}(n) \geq \rho .
$$

We see that the sequence of $B$-systems is just like our original sequence of systems, but has different parameters. (Recall that our original model does allow converging arrival rates per server, not just constant.)

\section{$7 \quad$ Some auxiliary facts}

Lemma 8. Let $a \in[0,1]$ be fixed. Consider a sequence of processes such that, for each $n$, at time 0 , we identify a subset, consisting of an servers. As the process evolves, for $t \geq 0$, we will keep track of those servers - let us call them "tagged." Denote by $f^{n}(t), t \geq 0$, the (scaled) number of the tagged servers, which are not selected by any new job arrival in the interval $[0, t]$. Then, for any fixed $t \geq 0$,

$$
(P) \liminf _{n \rightarrow \infty} f^{n}(t) \geq a e^{-\lambda d^{2} t} .
$$

Since, by definition, $f^{n}(t)$ is non-increasing in $t$, as a corollary of (14), we obtain the following stronger property: for any $t \geq 0$,

$$
(P) \liminf _{n \rightarrow \infty}\left(f^{n}(\tau), \tau \in[0, t]\right) \geq a e^{-\lambda d^{2} t} .
$$

Proof. Using coupling, we see that the stochastic lower bound $\hat{f}^{n}(t)$ of the process $f^{n}(t)$ can be obtained by considering the following "worst case" unaffected tagged set scenario: (a) each new job arrival selection set consists of $d$ servers and (b) if at least one of the selected servers is within the set of currently unaffected tagged servers, the latter set is reduced by $d$ servers. For the worst case unaffected tagged set, $\hat{f}(t)=a e^{-\lambda d^{2} t}$ is the deterministic mean-field (fluid) limit, solving $\hat{f}^{\prime}(t)=-(\lambda d) d \hat{f}(t)$ with $\hat{f}(0)=a$; here $\lambda d \hat{f}(t)$ is the (scaled, limiting) rate at which arriving jobs select a server within the set and $d$ is the number of servers removed upon each such event. Namely, using standard techniques ("large number of servers" fluid limit), cf. [11], it is straightforward to show that, for any $t \geq 0$,

$$
(P) \lim _{n \rightarrow \infty}\left(\hat{f}^{n}(\tau), \tau \in[0, t]\right)=(\hat{f}(\tau), \tau \in[0, t]),
$$

which then implies (14). 
Lemma 9. Let $a \in[0,1]$ and $h>0$ be fixed. Consider a sequence of processes $x^{n}(\cdot)$ with initial states $x^{n}(0)$ satisfying the following condition: the (scaled) number of servers with workload exactly equal to $h$, is at least a; namely, $x_{h-}^{n}(0)-x_{h}^{n}(0) \geq a$. Then,

$$
(P) \liminf _{n \rightarrow \infty}\left(x_{h-t-}^{n}(t)-x_{h-t}^{n}(t), 0 \leq t<h\right) \geq a e^{-\lambda d^{2} h} .
$$

(Informally, in words, "when $n$ is large, then with high probability a positive, bounded away from zero, jump in $x^{n}(t)$ "moves" left at speed 1 from initial point $\left.w=h.\right)$

Proof. Consider the servers with initial workload exactly equal to $h$ as tagged servers, and apply Lemma 8

\section{Asymptotic independence over a finite interval}

The constructions and the results in this section closely follow those in [5] (Section 7) and (to a lesser degree) in [9] (proofs of Lemmas 3.1 and 3.2). We give them here (along with proofs) in the setting/notation that we need for our model.

Suppose a finite set of fractions (a probability distribution) $a_{1}, a_{2}, \ldots, a_{K}$ is fixed, where all $a_{k}>0$ and $\sum_{k} a_{k}=1$. Also fixed is a set of numbers $w_{k} \in[0, \infty], k=1, \ldots, K$. Let the truncation parameter $c \in[0, \infty]$ be fixed. In this section, we consider a sequence of our systems, indexed by $n \rightarrow \infty$, with initial states such that $a_{k} n$ servers have workload exactly $w_{k}, k=1, \ldots, K$. Suppose, initially the server indices $1, \ldots, n$ are assigned in the order of a server set permutation chosen uniformly at random. This means, in particular, that $\mathbb{P}\left\{W_{1}^{n}(0)=w_{k}\right\}=a_{k}$.

We now formally construct a random process $U_{1}(t), t \geq 0$. Lemma 11 below will show that, for each $t$, $W_{1}^{n}(t) \Rightarrow U_{1}(t)$ as $n \rightarrow \infty$. So, informally speaking, this is a construction of the evolution of a server workload in a system with "infinite number of servers."

Fix $t \geq 0$. Suppose we consider a server, labeled 1 to be specific. Let $U_{1}(t)$ denote its workload at time $t$. Just like for our original system (with a finite number of servers), we will use the terminology of a job selection set, although here the latter will be defined formally, not as a result of an actual selection process. Denote $\alpha_{j}=\lambda_{j} d_{j}, \alpha=\sum_{j} \alpha_{j}$. Then, by definition, the job arrivals of type $j$ selecting server 1 occur according to an independent Poisson process of rate $\alpha_{j}$. We now define the dependence set $\bar{D}_{1}(t)$ of server 1 at time $t$. It is defined via a branching process, running in reverse time from $t$ to 0 ; the reverse time index is $0 \leq s \leq t$, with $s$ corresponding to actual (forward) time $t-s$. To improve the exposition, we will define the construction via an example, shown in Figure 1 in this example we also assume that there are two job classes, with $d_{1}=3$ and $d_{2}=2$. Ovals indicate job arrivals, with crosses showing the servers they select. By definition, set $A(s)$ at $s=0$ is $A(0)=\{1\}$. As $s$ increases, set $A(s)$ increases as follows. There is a Poisson process (in reversed time $s$ ) of rate $\alpha_{j}$, for each class $j$, of job arrivals selecting server 1 . Suppose first such arrival occurs at time $s_{1}$ (which corresponds to forward time $t_{1}=t-s_{1}$ shown in Figure 1), and it happens to be a class-2 job arrival. The set $A(s)$ remains unchanged in the interval $\left[0, s_{1}\right]$, and at time $s_{1}$ we add $d_{2}-1=1$ servers to the set, which we call "children" of node 1 added at time $s_{1}$; each added child server is a new server with distinct index - it is server 8 in Figure 1. So, $A\left(s_{1}+\right)=\{1,8\}$, if we adopt a (non-essential) convention that $A(s)$ is left-continuous. Starting time $s_{1}$, each server in $A(s)$ receives job arrivals according to independent Poisson processes, same as for server 1 . Let $s_{2}$ (corresponding to forward time $t_{1}=t-s_{1}$ in Figure 1) be the next job arrival time, to any of the servers in set $A(s)$; in Figure 1 it is class- 1 job arrival to server 8. Then $A(s)$ is constant in $\left(s_{1}, s_{2}\right]$, and at time $s_{2}$ we add $d_{1}-1=2$ new servers, with distinct indices 9,10 - these are children of server 8 added at $s_{2}$. After $s_{2}$ we consider independent Poisson job arrival processes to each of servers in $A(s)$. The next job arrival is time $s_{3}$ (corresponding to forward time $t_{3}=t-s_{3}$ in Figure 1), which happens to be of class 1 at server 1, which adds two new children, 2 and 3 , of server 1 at time $s_{3}$; and so on. In Figure 1 there are 8 job arrivals in total in $[0, t]$, and the final set $A(t)=\{1,2, \ldots, 13\}$. We emphasize that each added child server in this construction is a new server 


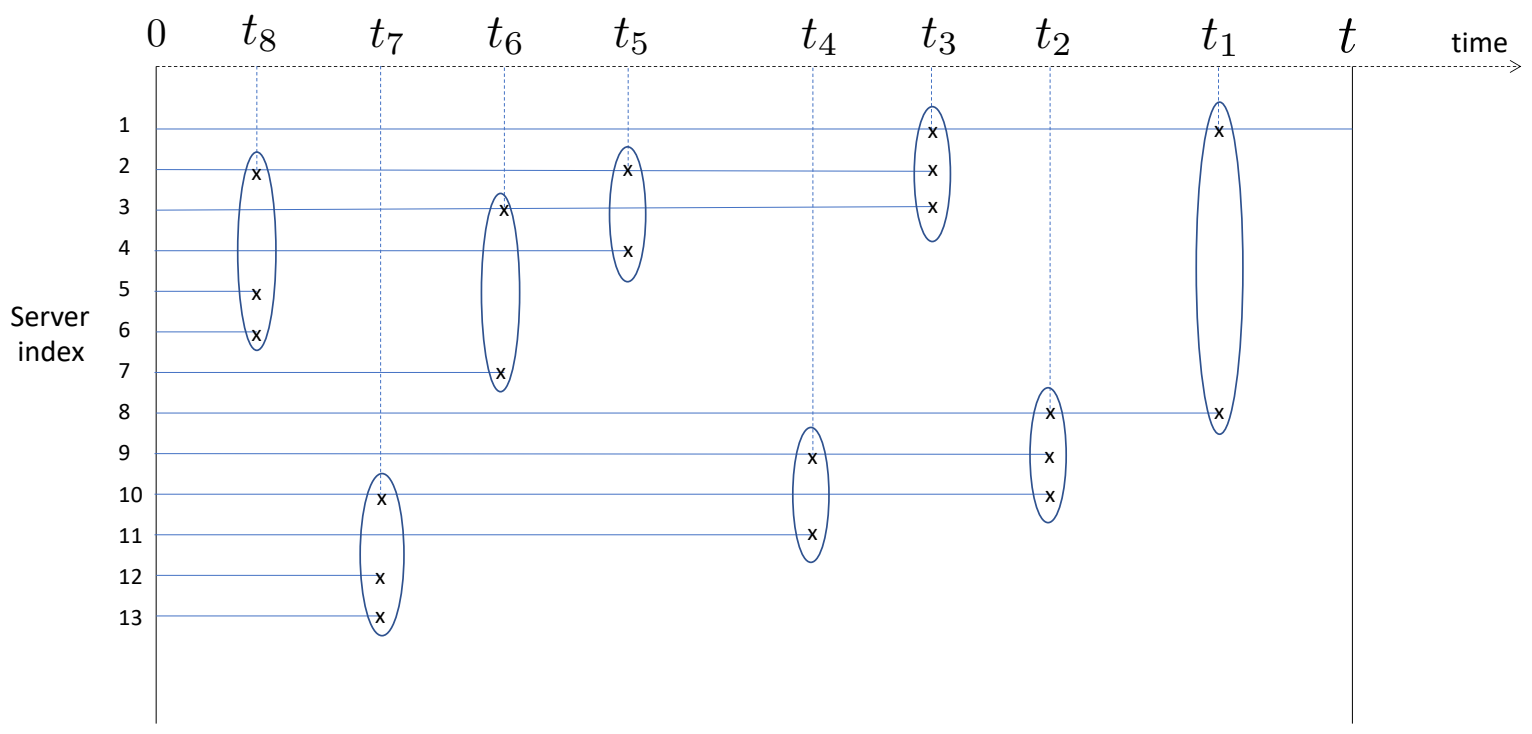

Figure 1: Construction of dependence set $\bar{D}_{1}(t)$.

with a distinct index. Then, by definition, $\bar{D}_{1}(t)=A(t)$. From now on, with a slight abuse of terminology, when we say "dependence set $\bar{D}_{1}(t)$," we assume that $\bar{D}_{1}(t)$ includes not only the set of servers, but also the graph describing the "parent-child" dependence structure and the job arrival times and classes. (For $\bar{D}_{1}(t)$, by construction, the graph describing the dependence structure is a tree.)

Now, given a realization of $\bar{D}_{1}(t)$, the random value of $U_{1}(t)$ is obtained by letting the initial workloads of all servers $i \in \bar{D}_{1}(t)$ to be i.i.d. with the distribution $\mathbb{P}\left\{U_{i}(0)=w_{k}\right\}=a_{k}, k=1, \ldots, K$, and with the component size vectors for the involved job arrivals being independent with the corresponding distributions. As usual, between the times of job arrivals selecting a server, the workload $U_{i}(t)$ of each server decreases at rate 1 (unless and until it reaches 0 ). Clearly, if a server $i \in \bar{D}_{1}(t)$ is added as a child at (forward) time $\tau$, the evolution of its workload after time $\tau$ does not affect the value of $U_{1}(t)$. This completes the definition of $U_{1}(t)$.

Lemma 10. For any $t \geq 0$, the (random) cardinality $f(t)=\left|\bar{D}_{1}(t)\right|$ of the dependence set $\bar{D}_{1}(t)$ is finite. Moreover, $f(t)$ satisfies

$$
f^{\prime}(t)=\gamma f(t), \quad \text { with } \gamma=\sum_{j} \alpha_{j}\left(d_{j}-1\right)
$$

and therefore

$$
\mathbb{E}\left|\bar{D}_{1}(t)\right|=e^{\gamma t}, t \geq 0
$$

Proof. The proof uses the branching process argument. (It is analogous to that used in Section 7 of [5].) Clearly, $f(s), s \geq 0$, can be equivalently viewed as the cardinality of set $A(s)$ in the definition of $D_{1}(t)$. In a small time interval $[s, s+\Delta s]$, the expected total number of all children that will be added is $f(s)\left[\sum_{j} \alpha_{j}\left(d_{j}-1\right) \Delta s+o(\Delta s)\right]$. This leads to ODE $[17)$. We omit further details.

Lemma 11. For any $t \geq 0$, as $n \rightarrow \infty$,

$$
W_{1}^{n}(t) \Rightarrow U_{1}(t)
$$

and, moreover,

$$
\mathbb{P}\left\{W_{1}^{n}(t)>w\right\} \rightarrow \mathbb{P}\left\{U_{1}(t)>w\right\} \quad \text { for any } w \geq 0
$$


Proof. Note that the workloads $W_{i}^{n}(t)$ are those of the servers $i=1, \ldots, n$, in the system with finite $n$. (Also recall that initially the servers' indices are assigned in a random order.) Let us define the dependence set $D_{i}^{n}(t)$ for server $i$ at time $t$, in the system with given $n$. The construction of $D_{i}^{n}(t)$ is analogous to the construction of $\bar{D}_{1}(t)$ for the "infinite system," except here, as we increase the set $A_{i}^{n}(s)$ (analogous to set $A(s)$ in the definition of $\left.\bar{D}_{1}(t)\right)$ in reversed time $s$, the children are added according to actual job arrivals in the finite system with $n$ servers. As a result: (a) a single job arrival may simultaneously select multiple servers in $A_{i}^{n}(s)$; (b) when children are added at time $s$, they may be servers already present in $A_{i}^{n}(s)$; (c) the graph describing the parent-child dependence of servers in $D_{i}^{n}(t)$ is not necessarily a tree.

Note that, marginally for each server $i$, the job arrival processes of different classes $j$ selecting this server are still independent Poisson processes with rates $\alpha_{j}$; however, the independence of these arrival processes across the servers no longer holds. On the other hand, consider any fixed $C>0$. Suppose there is a job arrival selecting at least one server in $A_{i}^{n}(s)$ at (reversed) time $s$. Consider event $E\left(A_{i}^{n}(s)\right)=\{$ The arriving job selects more than one server $A_{i}^{n}(s)$ or any of the children servers added by the arrival are already in $A_{i}^{n}(s)$. $\}$ Then, as $n \rightarrow \infty, \mathbb{P}\left\{E\left(A_{i}^{n}(s)\right)\right\} \rightarrow 0$, uniformly in all possible $A_{i}^{n}(s)$ with $\left|A_{i}^{n}(s)\right| \leq C$. This in turn implies that, as $n \rightarrow \infty$, the rate at which a class- $j$ job arrival selecting a server in $A_{i}^{n}(s)$ occurs, converges to $\left|A_{i}^{n}(s)\right| \alpha_{j}$, uniformly in $A_{i}^{n}(s)$ with $\left|A_{i}^{n}(s)\right| \leq C$.

Using the above observations, the proof proceeds by showing that, as $n \rightarrow \infty$, the set $D_{i}^{n}(t)$ converges to $\bar{D}_{1}(t)$, in an appropriate sense specified below. It is easy to see that, for all sufficiently large $n$,

$$
\left|D_{1}^{n}(t)\right| \leq_{s t}\left|\bar{D}_{1, \epsilon}(t)\right|
$$

where $\bar{D}_{1, \epsilon}(t)$ is constructed the same way as $\bar{D}_{1}(t)$, but with arrival rates $\lambda_{j}$ replaced by slightly larger rates $(1+\epsilon) \lambda_{j}, \epsilon>0$. By Lemma 10 .

$$
\mathbb{E}\left|\bar{D}_{1, \epsilon}(t)\right|=e^{\gamma^{\prime} t}, t \geq 0,
$$

where $\gamma^{\prime}=(1+\epsilon) \sum_{j} \alpha_{j}\left(d_{j}-1\right)$. We conclude that, uniformly in all sufficiently large $n,\left|D_{1}^{n}(t)\right|$ is stochastically dominated by a proper non-negative random variable. Using this fact, along with the observations in the previous paragraph, we can easily couple the constructions of $D_{1}^{n}(t)$ for each $n$ and the construction of $\bar{D}_{1}(t)$ in such a way that, w.p.1,

$$
D_{1}^{n}(t) \rightarrow \bar{D}_{1}(t), \quad W_{1}^{n}(t) \rightarrow U_{1}(t),
$$

where the $D_{1}^{n}(t) \rightarrow \bar{D}_{1}(t)$ is defined as follows. We say that (the realizations of) $D_{1}^{n}(t)$ and $\bar{D}_{1}(t)$ are equivalent, and write $D_{1}^{n}(t)=\bar{D}_{1}(t)$ if they are equal, up to relabeling of servers other than server 1 ; this means that they have equal sets of servers, equal number of job arrivals, equal parent-child dependence graph, and equal job arrival times. We say that the convergence $D_{1}^{n}(t) \rightarrow \bar{D}_{1}(t)$ holds (for the realizations of $D_{1}^{n}(t)$ and $\left.\bar{D}_{1}(t)\right)$ if $D_{1}^{n}(t)=\bar{D}_{1}(t)$ for all sufficiently large $n$. The convergence 21) proves (18). Moreover, clearly the coupling construction can be such that, w.p.1, for all sufficiently large $n$, we in fact have $W_{1}^{n}(t)=U_{1}(t)$; this proves 19 .

Let us denote:

$$
x_{w}^{c}(t) \doteq \mathbb{P}\left\{U_{1}(t)>w\right\} .
$$

Lemma 12. For the sequence of systems, considered in this section, the following holds for any $w \geq 0$ and $t \geq 0$ :

$$
x_{w}^{n, c}(t) \stackrel{P}{\rightarrow} x_{w}^{c}(t) .
$$

Proof. Consider a fixed $t$, a fixed $w$, and two fixed servers 1 and 2. Denote by $E_{i}^{n} \doteq\left\{W_{1}^{n}(t)>w\right\}, i=1,2$. To prove (23), it suffices to prove that the covariance of $\mathbf{I}\left(E_{1}^{n}\right)$ and $\mathbf{I}\left(E_{2}^{n}\right)$ vanishes, namely

$$
\mathbb{E} \mathbf{I}\left(E_{1}^{n}, E_{2}^{n}\right)-\mathbb{E} \mathbf{I}\left(E_{1}^{n}\right) \mathbb{E} \mathbf{I}\left(E_{2}^{n}\right) \rightarrow 0, \quad n \rightarrow \infty .
$$

Indeed, by the symmetry (exchangeability of the servers), from $(19)$ and 22 we have that $\mathbb{E} x_{w}^{n, c}(t)=$ $\mathbb{E} \mathbf{I}\left(E_{1}^{n}\right)=\mathbb{P}\left\{W_{1}^{n}(t)>w\right\} \rightarrow \mathbb{P}\left\{U_{1}(t)>w\right\}=x_{w}^{c}(t)$; then the vanishing covariance 24) implies that the variance of $x_{w}^{n, c}(t)$ vanishes as well. 
In turn, to prove 24 it suffices to prove

$$
\mathbb{E} \mathbf{I}\left(E_{1}^{n}, E_{2}^{n}\right) \rightarrow\left[\mathbb{P}\left\{U_{1}(t)>w\right\}\right]^{2}, \quad n \rightarrow \infty .
$$

This follows from a straightforward extension of the constructions we used in the proof of Lemma 11. Namely, consider two independent constructions of the "infinite server system" dependence sets $\bar{D}_{1}(t)$ and $\bar{D}_{2}(t)$; the latter is defined the same way as the former, except the "seed" server is 2 , not 1 ; WLOG we can assume that the sets of server indices in $\bar{D}_{1}(t)$ and $\bar{D}_{2}(t)$ do not intersect. Then consider the joint construction of the dependence sets $D_{1}^{n}(t)$ and $D_{2}^{n}(t)$, which starts with the "seed" set $A^{n}(0)=\{1,2\}$, corresponding to time $t$, and then goes "backwards in time." In this case $D_{1}^{n}(t)$ and $D_{2}^{n}(t)$ are not independent, but exactly the same argument as in the proof of Lemma 11 shows that a coupling exists such that, w.p.1, $\left(D_{1}^{n}(t), D_{2}^{n}(t)\right) \rightarrow$ $\left(\bar{D}_{1}(t), \bar{D}_{2}(t)\right)$ and $\left(W_{1}^{n}(t), W_{2}^{n}(t)\right) \rightarrow\left(U_{1}(t), U_{2}(t)\right.$. (The convergence $\left(D_{1}^{n}(t), D_{2}^{n}(t)\right) \rightarrow\left(\bar{D}_{1}(t), \bar{D}_{2}(t)\right)$ is defined the same way as $D_{1}^{n}(t) \rightarrow \bar{D}_{1}(t)$, except the equivalence $\left(D_{1}^{n}(t), D_{2}^{n}(t)\right)=\left(\bar{D}_{1}(t), \bar{D}_{2}(t)\right)$ is understood as equality up to relabeling of the servers other than 1 and 2.) Recall $\bar{D}_{1}(t)$ and $\bar{D}_{2}(t)$ (and $U_{1}(t)$ and $U_{2}(t)$ ) are independent. Moreover, the coupling can be such that, w.p.1, for all sufficiently large $n$, we in fact have $\left(W_{1}^{n}(t), W_{2}^{n}(t)\right)=\left(U_{1}(t), U_{2}(t)\right.$. This proves 25), and then the lemma.

\section{$9 \quad$ Fluid sample paths}

Suppose we are in the setting of Section 8 . Defined there function $x^{c}(t), t \geq 0$, with values in $\mathcal{X}$, we will call a fluid sample path (FSP). Clearly, an FSP initial state is: $x_{w}^{c}(0)=\sum_{k} a_{k} \mathbf{I}\left\{a_{k}>w\right\}, 0 \leq w<\infty$. (Note that an FSP, by definition, arises as a result of the limiting procedure specified in Section 8 . Namely, the initial states of the pre-limit systems are such that exactly a fraction $a_{k}$ of servers has workload exactly $w_{k}$, for some fixed parameters $a_{k}>0$ (such that $\sum_{k} a_{k}=1$ ) and $w_{k} \in[0, \infty]$. In this paper we will only need FSPs defined this way.)

By $x^{c, \emptyset}(t), t \geq 0$, we will denote the special FSP with $a_{1}=1, w_{1}=0$; this means that each pre-limit system starts from the "empty" initial state, with all initial workloads being 0 . Of course, $x_{0}^{c, \emptyset}(0)=0$. This is the FSP "starting from the empty initial state." As a special case of Lemma 12, we obtain that for any fixed $c \leq \infty, w \geq 0, t \geq 0$,

$$
x_{w}^{n, c, \emptyset}(t) \stackrel{P}{\rightarrow} x_{w}^{c, \emptyset}(t)
$$

The FSP definition and Lemma 3 imply the following monotonicity property for the FSPs.

Lemma 13. (i) Consider two FSPs, $x^{c}(\cdot)$ and $\hat{x}^{\hat{c}}(\cdot)$, such that $c \leq \hat{c}$ and $x^{c}(0) \leq \hat{x}^{\hat{c}}(0)$. Then $x^{c}(t) \leq \hat{x}^{\hat{c}}(t)$ for all $t \geq 0$. (ii) Consider two FSPs, $x(\cdot)$ and $\hat{x}(\cdot)$, such that, for some $0 \leq h<\infty$ and $\tau \geq 0, \hat{x}_{h}(0)=0$ and $x_{h}(\tau)>\hat{x}_{0}(0)$. Then, $\hat{x}(t) \leq x(\tau+t)$ for all $t \geq 0$.

\section{Properties of FSPs starting from the empty initial state}

In this section we study the properties of the FSPs $x_{w}^{c, \emptyset}(t), t \geq 0$, starting from the empty initial state. Recall that $c \in[0, \infty]$ is the truncation parameter. Note that an FSP $x_{w}^{c, \emptyset}(t), t \geq 0$, can be viewed as a scalar function of $(c, w, t)$.

Lemma 14. Function $x_{w}^{c, \emptyset}(t)$ is non-decreasing in $c, t$, and is non-increasing in $w$.

Proof. This follows from (26), along with Lemma 3 and Corollary 4 ,

Lemma 15. For any $c>0$ and $t>0, x^{c, \emptyset}(t)$ is proper and $x_{w}^{c, \emptyset}(t)>0$ for each $w<c$.

Proof. By definition $22, x_{w}^{c, \emptyset}(t)=\mathbb{P}\left\{U_{1}(t)>w\right\}$, where the construction of $U_{1}(t)$ is for the special case when all servers' workloads are initially 0 . It follows from the construction that $U_{1}(t)$ is finite w.p. 1 for any 
finite $t \geq 0$, that is $x^{c, \emptyset}(t)$ is proper. Moreover, it is easy to see from the construction that, for any $t \geq 0$ and $w<c$, the probability $\mathbb{P}\left\{U_{1}(t)>w\right\}$ must be positive.

Sometimes, as in the proof of the next lemma, it will be convenient to interpret a given server workload evolution as the movement of a "particle" in $[0, \infty]$, with the workload being the particle location. With this interpretation, between the times of job arrivals that select the server, the particle moves left at the constant speed 1 until/unless it "hits" 0. At the times when a new job arrival adds to the server workload, the particle "jumps right" by the distance equal to the added workload.

Lemma 16. As a function of $w \geq 0, x_{w}^{c, \emptyset}(t)$ is Lipschitz, uniformly in $c \leq \infty$ and $t \geq 0$.

Proof. Consider time $t$ and interval $[w, w+\delta]$. Consider the (pre-limit) system and process, with fixed $n$. All particles (server workloads) that are in $[w, w+\delta]$ at time $t$, at time $t+\delta$ will be in $[0, w]$, unless they are selected by new job arrivals in $[t, t+\delta]$. Recall that new jobs arrive as a Poisson process of (unscaled) rate $\lambda n$, for a given $n$, and each job selects at most $d$ particles. Let $g_{w}^{n}$ be the (scaled) number of particles that cross point $w$ from left to right in the interval $[t, t+\delta]$. By the law of large numbers,

$$
(P) \limsup _{n \rightarrow \infty} g_{w}^{n} \leq \lambda d \delta .
$$

Therefore,

$$
\begin{gathered}
x_{w}(t+\delta)-x_{w}(t)=(P) \lim _{n \rightarrow \infty}\left[x_{w}^{n}(t+\delta)-x_{w}^{n}(t)\right] \leq \\
-(P) \lim _{n \rightarrow \infty}\left[x_{w}^{n}(t)-x_{w+\delta}^{n}(t)\right]+(P) \limsup _{n \rightarrow \infty} g_{w}^{n} \leq-\left[x_{w}(t)-x_{w+\delta}(t)\right]+\lambda d \delta .
\end{gathered}
$$

But, $0 \leq x_{w}(t+\delta)-x_{w}(t)$. Therefore, $x_{w}(t)-x_{w+\delta}(t) \leq \lambda d \delta$.

Lemma 17. For any fixed $0 \leq w<\infty$, function $x_{w}^{\emptyset}(t), t \geq 0$, is strictly increasing in $t$. (Note that here we consider specifically the non-truncated system, $c=\infty$.)

Proof. Fix any $\tau \geq 0$, any $\delta>0$, and any $w \geq 0$. We will show that $x_{w}^{\emptyset}(\tau+\delta)>x_{w}^{\emptyset}(\tau)$. Suppose, first, that $w>0$. By Lemma 15 at time $\delta, x^{\emptyset}(\delta)$ is such that $x_{u}^{\emptyset}(\delta)$ is positive for all $u<\infty$. Consider a state, let us denote it $\hat{x}(\delta)$, such that $\hat{x}_{u}(\delta)=a \in(0,1)$ for $u<w+\tau$, and $\hat{x}_{u}(\delta)=0$ for $u \geq w+\tau$. (Fraction $a$ of "servers" have workload exactly $w+t$, while the rest of the "servers" have workload 0 .) We can and do pick $a>0$ small enough so that $x_{u}^{\emptyset}(\delta)>\hat{x}_{u}(\delta)$ for all $u \geq 0$. For each $n$, let us consider the process $x_{u}^{n, \emptyset}(\cdot)$ in the time interval $[\delta, \infty)$, and compare it with the process $\hat{x}^{n}(\cdot)$ in the same time interval, starting from state $\hat{x}^{n}(\delta)=\hat{x}(\delta)$. We have that

$$
\lim _{n \rightarrow \infty} \mathbb{P}\left\{x_{u}^{n, \emptyset}(\delta) \geq \hat{x}_{u}^{n}(\delta), \forall u \geq 0\right\}=1 .
$$

Given this, and using the monotonicity, we can couple these processes in a way such that

$$
\lim _{n \rightarrow \infty} \mathbb{P}\left\{x_{u}^{n, \emptyset}(\delta+t) \geq \hat{x}_{u}^{n}(\delta+t), \forall u \geq 0, \forall t \geq 0\right\}=1
$$

By Lemma 12 , for any $t \geq 0$ and $u \geq 0, \hat{x}_{u}^{n}(\delta+t) \stackrel{P}{\rightarrow} \hat{x}_{u}(\delta+t)$, where, by monotonicity, $\hat{x}_{u}(\delta+t) \geq x_{u}^{\emptyset}(t)$. Now, by Lemma 9 .

$$
(P) \liminf _{n \rightarrow \infty}\left(\hat{x}_{w+\tau-t-}^{n}(\delta+t)-\hat{x}_{w+\tau-t}^{n}(\delta+t), 0 \leq t<w+\tau\right) \geq a e^{-\lambda d^{2}(w+\tau)}=\epsilon>0 .
$$

This implies that, for any $0 \leq t<w+\tau$,

$$
\hat{x}_{w+\tau-t-}(\delta+t)-\hat{x}_{w+\tau-t}(\delta+t) \geq \epsilon,
$$

and then

$$
\hat{x}_{w+\tau-t-}(\delta+t)-x_{w+\tau-t}^{\emptyset}(t) \geq \epsilon,
$$


and finally

$$
x_{w+\tau-t}^{\emptyset}(\delta+t)-x_{w+\tau-t}^{\emptyset}(t) \geq \epsilon .
$$

In particular, substituting $t=\tau$, we obtain $x_{w}^{\emptyset}(\delta+\tau)-x_{w}^{\emptyset}(\tau) \geq \epsilon>0$, which completes the proof for the case $w>0$. To treat the case $w=0$, observe that the proof of (27) in fact holds as is, with the same $\epsilon>0$ in the RHS, if in the LHS we replace $w$ by any $u \in(0, w]$. Therefore, $x_{u}^{\emptyset}(\delta+\tau)-x_{u}^{\emptyset}(\tau) \geq \epsilon>0$ for all small positive $u>0$, and then, by the continuity of $x_{u}^{\emptyset}(\tau)$, for $u=0$ as well.

As a corollary from the results in this section, we obtain the following uniform convergence to an FSP starting from the empty initial state.

Lemma 18. For any $t \geq 0,\left\|x^{n, c, \emptyset}(t)-x^{c, \emptyset}(t)\right\| \stackrel{P}{\rightarrow} 0$.

\section{Fixed point}

Given the properties of the FSPs derived above, we see that, as $t \rightarrow \infty$,

$$
x^{c, \emptyset}(t) \stackrel{\text { u.o.c. }}{\rightarrow} x^{*, c} \in \mathcal{X} .
$$

The element $x^{*, c} \in \mathcal{X}$ we will call the fixed point (for a given $c$ ). In particular, $x^{*}=x^{*, \infty}$.

Given that all functions $x_{w}^{c, \emptyset}(t), w \geq 0$, are uniformly Lipschitz, their u.o.c. limits $x_{w}^{*, c}, w \geq 0$, are uniformly Lipschitz as well.

Lemma 19. The fixed point $x^{*, c}$ is such that $x_{0}^{*, c} \leq \lambda$.

Proof. The proof is by contradiction. Suppose not, i.e. $x_{0}^{*, c}>\lambda$. Choose $T>0$ sufficiently large, so that

$$
(1 / T) \int_{0}^{T} x_{0}^{c, \emptyset}(t) d t>\lambda .
$$

Note that, for any $n$,

$$
(1 / T) \int_{0}^{T} \mathbb{E} x_{0}^{n, c, \emptyset}(t) d t \leq \lambda,
$$

because the LHS is the expected amount of work (per server per unit time) processed by the system in $[0, T]$ - it cannot exceed $\lambda$, which is the expected amount of work (per server per unit time) arrived into the system in $[0, T]$. But we have

$$
\lim _{n \rightarrow \infty}(1 / T) \int_{0}^{T} \mathbb{E} x_{0}^{n, c, \emptyset}(t) d t=(1 / T) \int_{0}^{T} x_{0}^{c, \emptyset}(t) d t>\lambda
$$

This contradiction completes the proof.

Lemma 20. For any $w \in[0, \infty)$, as $c \uparrow \infty, x_{w}^{*, c} \uparrow x_{w}^{*}$.

Proof. It follows from Lemma 5, that the processes with a finite $c$ and $c=\infty$ can be coupled so that $x_{w}^{n, c, \emptyset}(t)=x_{w}^{n, \emptyset}(t)$ for $t<c-w$. Therefore, $x_{w}^{c, \emptyset}(t)=x_{w}^{\emptyset}(t)$ for $t<c-w$. Then, for any $\epsilon>0$, we can choose a sufficiently large $t$ and then sufficiently large $c$, so that

$$
x_{w}^{c, \emptyset}(t)=x_{w}^{\emptyset}(t)>x_{w}^{*}-\epsilon,
$$

and then $x_{w}^{*, c}>x_{w}^{*}-\epsilon$.

In particular, from Lemma $20, x_{0}^{*, c} \uparrow x_{0}^{*}$ as $c \uparrow \infty$.

Note that, for a finite $c$, the fixed point $x^{*, c}$ is automatically proper, since $x_{c}^{*, c}=0$. Our next goal is to show that $x^{*}$ is proper, i.e. $x_{w}^{*} \downarrow 0$ as $w \rightarrow \infty$, and $x_{0}^{*}=\lambda$. 
Lemma 21. If fixed point $x^{*}$ is proper, then $x_{0}^{*}=\lambda$.

Proof. Fix any $\epsilon>0$ and any $\epsilon^{\prime}>0$. Then fix a large $h>0$ such that

$$
1-\left(1-x_{h}^{*}\right)^{d}<\epsilon^{\prime}
$$

if we have a subset consisting of $\left(1-x_{h}^{*}\right) n$ servers, then the probability that an arriving job selects a server outside this set is less than $\epsilon^{\prime}$. Finally, fix a sufficiently large finite $c>h$ so that if a job selects only servers with workloads at most $h$, then the expected amount of (this job's) work lost due to truncation is less than $\epsilon$.

For each $n$, consider process $x^{n, c, \emptyset}(\cdot)$, with the chosen above truncation parameter $c$. For any $t \geq 0$, since $x_{h}^{c, \emptyset}(t) \leq x_{h}^{\emptyset}(t)<x_{h}^{*}$,

$$
\lim _{n \rightarrow \infty} \mathbb{P}\left\{1-\left(1-x_{h}^{n, c, \emptyset}(t)\right)^{d}<\epsilon^{\prime}\right\}=1 .
$$

Let $g_{\text {lost }}^{n}(T)$ denote the expected total (scaled) amount of workload lost due to truncation in the interval $[0, T]$. Recall that the job arrivals process is Poisson. Then,

$$
\limsup _{n \rightarrow \infty} g_{\text {lost }}^{n}(T)\left[\lambda \epsilon^{\prime}+\lambda\left(1-\epsilon^{\prime}\right) \epsilon\right] T \leq \lambda\left(\epsilon^{\prime}+\epsilon\right) T .
$$

Let $g_{\text {out }}^{n}(T)$ denote the expected total (scaled) amount of workload processed (and left the system) in the interval $[0, T]$.

$$
\lim _{n \rightarrow \infty} g_{\text {out }}^{n}(T)=\lim _{n \rightarrow \infty} \int_{0}^{T} \mathbb{E} x_{0}^{n, c, \emptyset}(t) d t=\int_{0}^{T} x_{0}^{c, \emptyset}(t) d t .
$$

Recall that the process starts from the empty state, so by work conservation, for any $T>0$,

$$
g_{\text {out }}^{n}(T) \geq \lambda T-g_{\text {lost }}^{n}(T)-c .
$$

We obtain

$$
\frac{1}{T} \int_{0}^{T} x_{0}^{c, \emptyset}(t) d t \geq \lambda\left(1-\epsilon^{\prime}-\epsilon\right)-c / T .
$$

Letting $T \rightarrow \infty$, and recalling that $\lim _{t \rightarrow \infty} x_{0}^{c, \emptyset}(t)=x_{0}^{*, c}$,

$$
x_{0}^{*, c} \geq \lambda\left(1-\epsilon^{\prime}-\epsilon\right) .
$$

Recall that such $c$ can be chosen for arbitrarily small $\epsilon^{\prime}>0$ and $\epsilon>0$. Then $x_{0}^{*}=\lim _{c} x_{0}^{*, c} \geq \lambda$. And by Lemma $\left[9, x_{0}^{*} \leq \lambda\right.$.

We now make the following observation. Up to this point in the paper (except in the statements of the main results, Theorem 1 and Corollary 2), we never used the condition $\lambda<1$. In particular, the definition of the fixed point $x^{*, c}$ does not depend on the condition $\lambda<1$ and neither do the statements and proofs of Lemmas 19 and 21. Using this fact, we obtain the following corollary from Lemmas 19 and 21. which will be used later.

Corollary 22. Consider the dependence of the fixed point $x^{*}$ on $\lambda$. If for a given $\lambda$ the fixed point $x^{*}$ is proper, then $x_{0}^{*}=\lambda$ (and then necessarily $\lambda \leq 1$ ).

It is not difficult to strengthen Corollary 22 to show that if $x^{*}$ is proper, then necessarily $x_{0}^{*}=\lambda<1$. But we will not need this fact.

From now on, some of our results/proofs do require that $\lambda<1$.

Lemma 23. Function $x_{w}^{*, c}, w \geq 0$, is strictly decreasing for $0 \leq w<c$. (This does require that $\lambda<1$.) 
Proof. The proof is by contradiction. Let $u \geq 0$ be a point such that $x_{w}^{*, c}$ is flat in an interval to the right of $u$, i.e. $x_{u}^{*, c}=x_{v}^{*, c}=a$ for some $u<v<c$. Note that $a \leq x_{0}^{*, c} \leq \lambda<1$. Pick any $\epsilon>0$, and then $t>0$ large enough, so that both $x_{u}^{c, \emptyset}(t)$ and $x_{v}^{c, \emptyset}(t)$ are in $(a-\epsilon, a)$. Pick any $0<b<1-\lambda$. At time $t$ let us consider the non-intersecting sets: $\bar{B}=\bar{B}(n)$ is the set of empty servers, $A=A(n)$ is the set of servers with workload greater than $v$. For their (scaled) cardinalities, we know that

$$
\begin{aligned}
& \lim _{n \rightarrow \infty} \mathbb{P}\left\{1-x_{0}^{n, c, \emptyset}(t)>b\right\}=1, \\
& \lim _{n \rightarrow \infty} \mathbb{P}\left\{x_{v}^{n, c, \emptyset}(t)>a-\epsilon\right\}=1 .
\end{aligned}
$$

Note that every server in set $A$ will have a workload greater than $u$ at time $t+(u-v)$. If event $\bar{B} \geq b n$ does hold at time $t$, let us pick a fixed subset $B=B(n)$ of $b n$ servers that are empty at $t$, and let us keep track of the servers in set $B$ over the time interval $[t, t+(v-u)]$. By Lemma 3 the distribution of workloads within this set stochastically dominates that of the following process: we consider only the servers in $B$ and we "ignore" any job arrival which selects at least one server outside $B$. Such a lower bounding process has the same structure as our original process, except it has a smaller number of servers, $b n$, and the job type arrival rates per server, $\lambda_{j}^{\prime}$, are different. Applying Lemma 15 and 26 to the lower bounding process, we obtain the following property. Denote by $g^{n}$ the (scaled) number of servers in $B$, which at time $t+(v-u)$ have workloads greater than $u$. Then, there exists $\delta>0$ such that

$$
\lim _{n \rightarrow \infty} \mathbb{P}\left\{g^{n}>\delta\right\}=1 .
$$

Combining these estimates, we obtain that

$$
\lim _{n \rightarrow \infty} \mathbb{P}\left\{x_{u}^{n, c, \emptyset}(t+(v-u))>a-\epsilon+\delta\right\}=1 .
$$

But, this is true for any $\epsilon>0$. So, we must have $x_{u}^{c, \emptyset}(t+(v-u))>a$, and then $x_{u}^{*, c}>a$, a contradiction.

Lemma 24. Fixed point $x^{*}$ is proper and $x_{0}^{*}=\lambda$. (This does require that $\lambda<1$.)

Proof. Given Lemma21 (or Corollary 22), it suffices to prove that $x^{*}$ is proper. Suppose not, i.e. $x_{w}^{*} \downarrow=a>0$ as $w \rightarrow \infty$. Then we claim the following. Consider the FSP $\hat{x}(t)$ corresponding to the initial state with fraction $a$ of servers having initially infinite workload and the remaining fraction $b=1-a$ of servers being empty. (This is the system described in Section 6.2. with the B-subsystem being initially empty.) So, $\hat{x}_{w}(0)=a$ for all $w \geq 0$. We claim that

$$
\hat{x}_{w}(t) \uparrow x_{w}^{*}, \forall w \geq 0 .
$$

(Consequently, $\left\|\hat{x}(t)-x^{*}\right\| \rightarrow 0$.) Note that by Lemma 13, $\hat{x}(t) \geq x^{\emptyset}(t)$, so $\lim _{t} \hat{x}_{w}(t) \geq x_{w}^{*}$. Then, to prove (28), it suffices to show that for any $t \geq 0, \hat{x}_{w}(t) \leq x_{w}^{*}$. For this, we consider the family of FSPs, $\hat{x}^{(h)}(\cdot)$, parameterized by $h<\infty$, corresponding to initial states such that the fraction $a$ of servers have initial workloads $h$ and the remaining fraction $b=1-a$ of servers are empty. For any fixed $h$, there exists a sufficiently large $\tau>0$ such that $x^{\emptyset}(\tau)>\hat{x}^{(h)}(0)$. (Here we use the fact that $x_{w}^{*}>a$ for all finite $w \geq 0$, because $x_{w}^{*}$ is strictly decreasing in $w$.) Then, by Lemma $13, \hat{x}^{(h)}(t) \leq x^{\emptyset}(\tau+t)<x^{*}$ for all $t \geq 0$. It remains to notice that for any fixed $w \geq 0$ and $t \geq 0$, if we choose $h>w+t$, then $\hat{x}_{w}(t)=\hat{x}_{w}^{(h)}(t)$. Thus, claim $(28)$ is proved.

Denote by $\bar{x}^{*}$ the element of $\mathcal{X}$, defined by $\bar{x}_{w}^{*}=\left(x_{w}^{*}-a\right) / b, w \geq 0$. By this definition, $\bar{x}^{*}$ is proper. Claim (28) proves that $\bar{x}^{*}$ is nothing else but the fixed point for the $B$-subsystem, as defined in Section 6.2, starting from the empty state. Since $\bar{x}^{*}$ is proper, by Corollary 22, $\bar{x}_{0}^{*}=\rho_{B}$. By (13), $\rho_{B} \geq \rho=\lambda$. But then $x_{0}^{*}=a+b \bar{x}_{0}^{*} \geq a+b \lambda>\lambda$. This contradicts Lemma 19 . 


\section{Proof of Theorem 1}

Consider the sequence $x^{n}(\infty)$. The sequence of their distributions is tight because $\mathcal{X}$ is compact. Consider any fixed subsequence along which $x^{n}(\infty) \Rightarrow x^{* *}$, where $x^{* *}$ is a random element in $\mathcal{X}$. It will suffice to show that, $x^{* *}=x^{*}$.

Lemma 25. $x^{*} \leq_{s t} x^{* *}$.

Proof. We can construct a stationary version of the process, $x^{n}(\cdot)$, and the process $x^{n, \emptyset}(\cdot)$ on a common probability space, so that $x_{w}^{n, \emptyset}(t) \leq x_{w}^{n}(t)$ for all $w$ and $t$. We conclude that $x^{n, \emptyset}(t) \leq_{s t} x^{n}(t)$ for any $t \geq 0$. Recall that $\left\|x^{n, \emptyset}(t)-x^{\emptyset}(t)\right\| \stackrel{P}{\rightarrow} 0$ as $n \rightarrow \infty$. This implies that, for any fixed $h>0$ and $t \geq 0$,

$$
(P) \liminf _{n \rightarrow \infty}\left(x_{w}^{n}(\infty), w \in[0, h]\right) \geq\left(x_{w}^{\emptyset}(t), w \in[0, h]\right) .
$$

Since this is true for any $t$, and $x_{w}^{\emptyset}(t)$ is strictly increasing in $t$, we conclude that, for any fixed $h>0$ and $t \geq 0$ the stronger property holds:

$$
\lim _{n \rightarrow \infty} \mathbb{P}\left\{x_{w}^{n}(\infty) \geq x_{w}^{\emptyset}(t), \forall w \in[0, h)\right\}=1 .
$$

Recalling that $x_{w}^{\emptyset}(t)$ is continuous in $w$, observe that the subset $\left\{y \in \mathcal{X} \mid y_{w} \geq x_{w}^{\emptyset}(t), \forall w \in[0, h)\right\}$ is closed. Therefore,

$$
\mathbb{P}\left\{x_{w}^{* *} \geq x_{w}^{\emptyset}(t), \forall w \in[0, h)\right\} \geq \underset{n}{\lim \sup } \mathbb{P}\left\{x_{w}^{n}(\infty) \geq x_{w}^{\emptyset}(t), \forall w \in[0, h)\right\}=1 .
$$

Thus, for any fixed $h>0$ and $t \geq 0$,

$$
\mathbb{P}\left\{x_{w}^{* *} \geq x_{w}^{\emptyset}(t), \forall w \in[0, h)\right\}=1 .
$$

It remains to recall that $x_{w}^{\emptyset}(t) \uparrow x_{w}^{*}$ as $t \rightarrow \infty$, to finally conclude that

$$
\mathbb{P}\left\{x_{w}^{* *} \geq x_{w}^{*}, \forall w \geq 0\right\}=1 .
$$

For future reference, note that Lemma 25 (or 290 ) implies, in particular,

$$
(P) \liminf _{n \rightarrow \infty} x_{0}^{n}(\infty) \geq x_{0}^{*} .
$$

Lemma 26. $x^{* *}=x^{*}$.

Proof. Suppose not, i.e. there exists $w>0$ and $a>0$ such that

$$
\mathbb{P}\left\{x_{w}^{* *}>x_{w}^{*}+a\right\}=\delta>0 .
$$

We will show that this leads to a contradiction. Let $x^{n}(\cdot)$ denote a stationary version of the process. The subset $\left\{y \in \mathcal{X} \mid y_{w}>x_{w}^{*}+a\right\}$ is open, so

$$
\liminf _{n} \mathbb{P}\left\{x_{w}^{n}(\infty)>x_{w}^{*}+a\right\} \geq \delta .
$$

Pick a sufficiently small $\epsilon>0$, such that $\epsilon<a e^{-\lambda d^{2} w}$. Pick a sufficiently large $\tau>0$ and then a sufficiently small $u>0$, such that $u \leq w$ and $x_{u}^{\emptyset}(\tau)+\epsilon>x_{0}^{*}+\epsilon / 2$. Let $\hat{x}^{n}(t) \doteq x^{n, \emptyset}(\tau+t)$, so that $\hat{x}^{n}(0)$ is equal in distribution to $x^{n, \emptyset}(\tau)$. Since $x_{v}^{\emptyset}(\tau)<x_{v}^{*}$ for all $v \leq w$, we know from the argument in the proof of Lemma 25, that, if we take independent initial distributions of $x^{n}(\cdot)$ and $\hat{x}^{n}(\cdot)$ then

$$
\lim _{n} \mathbb{P}\left\{x_{v}^{n}(0) \geq \hat{x}_{v}^{n}(0), \forall v \leq w\right\}=1 .
$$


Let us couple $x^{n}(\cdot)$ and $\hat{x}^{n}(\cdot)$ in the natural way. By Lemma 6, if condition $\left\{x_{v}^{n}(0) \geq \hat{x}_{v}^{n}(0), \forall v \leq w\right\}$ does hold, then condition $\left\{x_{v}^{n}(t) \geq \hat{x}_{v}^{n}(t), \forall v \leq w-t, \forall t \leq w\right\}$ holds as well. Furthermore, coupled with these processes, let us consider the following further modification $\tilde{x}^{n}(\cdot)$ of the process $x^{n}(\cdot)$. The initial state $x^{n}(0)$ is replaced by the initial state $\tilde{x}^{n}(0)$, where

$$
\begin{aligned}
& \tilde{x}_{u}^{n}(0)=x_{u}^{n}(0), u<w, \\
& \tilde{x}_{u}^{n}(0)=\hat{x}_{u}^{n}(0), u \geq w .
\end{aligned}
$$

Note that $\tilde{x}_{w-}^{n}(0)-\tilde{x}_{w}^{n}(0) \geq x_{w}^{n}(0)-\tilde{x}_{w}^{n}(0)$. Therefore, $\tilde{x}^{n}(0)$ is such that

$\liminf _{n} \mathbb{P}\{$ At least (scaled) number $a$ of servers have workload exactly $w\} \geq \delta$.

Consider the three coupled processes over the time interval $t \in[0, w-u]$. We can make the following conclusions:

$$
(P) \liminf _{n \rightarrow \infty} \hat{x}_{u}^{n}(w-u) \geq x_{u}^{\emptyset}(\tau+w-u)>x_{u}^{\emptyset}(\tau)
$$

which implies

$$
(P) \liminf _{n \rightarrow \infty} \tilde{x}_{u}^{n}(w-u)>x_{u}^{\emptyset}(\tau) .
$$

We also can use Lemma 16 to conclude that

$$
\liminf _{n} \mathbb{P}\left\{\tilde{x}_{u-}^{n}(w-u)-\tilde{x}_{u}^{n}(w-u) \geq \epsilon\right\} \geq \delta .
$$

Recalling our choice of $\epsilon$ and $u$, we obtain that

$$
\liminf _{n} \mathbb{P}\left\{\tilde{x}_{u-}^{n}(w-u) \geq x_{0}^{*}+\epsilon / 2\right\} \geq \delta .
$$

We also have

$$
\lim _{n} \mathbb{P}\left\{x_{u-}^{n}(w-u) \geq \tilde{x}_{u-}^{n}(w-u)\right\}=1 .
$$

The last two displays imply that

$$
\liminf _{n} \mathbb{P}\left\{x_{0}^{n}(w-u) \geq x_{0}^{*}+\epsilon / 2\right\} \geq \delta .
$$

Recall that $x^{n}(\cdot)$ is the stationary version of the process. Then,

$$
\liminf _{n} \mathbb{P}\left\{x_{0}^{n}(\infty) \geq x_{0}^{*}+\epsilon / 2\right\} \geq \delta .
$$

From this display and 30 ,

$$
\liminf _{n} \mathbb{E} x_{0}^{n}(\infty) \geq x_{0}^{*}+\delta \epsilon / 2>x_{0}^{*}=\lambda,
$$

which contradicts the conservation law $\mathbb{E} x_{0}^{n}(\infty)=\lambda$.

Lemmas 25 and 26 imply that $x^{n}(\infty) \Rightarrow x^{*}$, thus proving Theorem 1 .

\section{References}

[1] I. Adan, I. Kleiner, R. Righter, and G. Weiss. Fcfs parallel service systems and matching models. Performance Evaluation, 127:253-272, 2018.

[2] U. Ayesta, T. Bodas, and I. M. Verloop. On a unifying product form framework for redundancy models. Performance Evaluation, 127:93-119, 2018.

[3] U. Ayesta, T. Bodas, and I. M. Verloop. On redundancy-d with cancel-on-start aka join-shortest-work (d). ACM SIGMETRICS Performance Evaluation Review, 46(2):24-26, 2018. 
[4] M. Bramson. Stability of join the shortest queue networks. The Annals of Applied Probability, 21:1568$1625,2011$.

[5] M. Bramson, Y. Lu, and B. Prabhakar. Asymptotic independence of queues under randomized load balancing. Queueing Systems, 71:247-292, 2012.

[6] S. Foss and N. Chernova. On the stability of a partially accessible multi-station queue with statedependent routing. Queueing Systems, 29:55-73, 1998.

[7] K. Gardner, M. Harchol-Balter, A. Scheller-Wolf, M. Velednitsky, and S. Zbarsky. Redundancy-d: The power of d choices for redundancy. Operations Research, 65(4):1078-1094, 2017.

[8] K. Gardner, S. Zbarsky, S. Doroudi, M. Harchol-Balter, and E. Hyytia. Reducing latency via redundant requests: Exact analysis. ACM SIGMETRICS Performance Evaluation Review, 43(1):347-360, 2015.

[9] A. Greenberg, V. Malyshev, and S. Popov. Stochastic model of massively parallel computation. Markov Processes and Related Fields, 2:473 - 490, 1997.

[10] T. Hellemans, T. Bodas, and B. Van Houdt. Performance analysis of workload dependent load balancing policies. Proceedings of the ACM on Measurement and Analysis of Computing Systems, 3(2):35, 2019.

[11] G. Pang, R. Talreja, and W. Whitt. Martingale proofs of many-server heavy-traffic limits for markovian queues. Probability Surveys, 4:193-267, 2007.

[12] N. B. Shah, K. Lee, and K. Ramchandran. When do redundant requests reduce latency? IEEE Transactions on Communications, 64(2):715-722, 2015.

[13] A. L. Stolyar. Pull-based load distribution in large-scale heterogeneous service systems. Queueing Systems, 80(4):341-361, 2015.

[14] A. L. Stolyar. Pull-based load distribution among heterogeneous parallel servers: the case of multiple routers. Queueing Systems, 85(1-2):31-65, 2017.

[15] A. Vulimiri, P. Godfrey, R. Mittal, J. Sherry, S. Ratnasamy, and S. Shenker. Low latency via redundancy. CoNEXT 2013 - Proceedings of the 2013 ACM International Conference on Emerging Networking Experiments and Technologies, pages 283-294. Association for Computing Machinery, Jan. 2013.

[16] N. Vvedenskaya, R. Dobrushin, and F. Karpelevich. Queueing system with selection of the shortest of two queues: an asymptotic approach. Problems of Information Transmission, 32(1):20-34, 1996. 